\title{
Von Kinderdomänen zum Reich der Normen
}

\author{
Überlegungen zu Spielplätzen in Mexiko-Stadt und globale Perspektiven
}

Tuline Gülgönen

\begin{abstract}
In verschiedenen städtischen Kontexten weltweit brachte das 20. Jahrhundert den Übergang von der Stadt-als-Spielplatz, die von Kindern angeeignet und gelebt wird, zum Rückzug der Kinder auf speziell für sie gestaltete Spielplätze. Die gegenwärtige Verbreitung von Spielgeräten ähnlicher Bauart und Materialien in vielen Städten entspricht bestimmten Vorstellungen sowohl der Kindheit als auch der Stadt. Dieser Beitrag wählt einen interdisziplinären Ansatz, um diese Repräsentationen von Kindheit und Stadt und die Interaktion zwischen ihnen zu diskutieren. Mit besonderem Fokus auf Spielplätze in Mexiko-Stadt diskutiert der Aufsatz das Verhältnis zwischen diesen und der Entstehung eines globalen Bildes von Kindheit. Es wird untersucht, inwieweit der Bau von Spielplätzen tatsächlich auf die Förderung des kindlichen Spiels abzielt und ob Spielplätze heute noch räumliche Bezüge und Territorien für Kinder darstellen (können). Der Artikel setzt sich kritisch mit dem Begriff des öffentlichen Raums auseinander und zeigt, wie dieser dazu dienen kann, eine neoliberale Transformation der Stadt voranzutreiben.
\end{abstract}

Ersteinreichung: 28. Januar 2021; Veröffentlichung online: 26. November 2021

An English abstract can be found at the end of the document.

\begin{abstract}
„Ist doch das Kind kein Robinson, sind doch auch Kinder keine abgesonderteGemeinschaft, sondern ein Teil des Volkes und der Klasse, aus der sie kommen. So gibt denn auch ihr Spielzeug nicht von einem autonomen Sonderleben Zeugnis, sondern ist stummer Zeichendialog zwischen ihm und dem Volk. "(Benjamin 1969: 65)
\end{abstract}

\section{Einleitung}

Forschungen zu Geographien der Kindheit haben die große Bedeutung der Alltagsräume von Kindern hervorgehoben, in denen deren Identitäten und Leben geprägt werden und die sie gleichzeitig als Orte aktiv gestalten (Holloway/Valentine 2000; Rasmussen/Smidt 2003). Dem Spiel sowie der Art und Weise, auf die diese Räume das Spiel ermöglichen oder einschränken, wurden aufgrund ihrer Bedeutung im Leben von Kindern besondere Aufmerksamkeit zuteil - insbesondere, weil das Spiel der erste Mechanismus ist, durch den Kinder mit ihrer Umwelt vertraut werden (Matthews 1992; Murnaghan 2019). Dabei hat die sukzessive Verbannung 
der Kinder (oder bestimmter Gruppen von Kindern) von den Straßen, diein verschiedenen urbanen Kontexten weltweit beobachtbar ist, Spielplätze zu einem zentralen Raum im Leben vieler Stadtkinder werden lassen - und zu einem privilegierten Studienobjekt der Kindheitsgeographien.

In diesem Artikel möchteich einigezentrale Facetten von Spielplätzen und ihrer zeitlichen Transformation aufzeigen. Dazu fokussiere ich insbesondere auf den Kontext von Mexiko-Stadt. Ich unterscheide zwischen Orten für Kinder (places for children) - für Kinder gebauten Orte- und Orten der Kinder (children's places) - von Kindern angeeigneteOrte(Rasmussen 2004). Dabei geheich der Frage nach, inwieweit die von Erwachsenen gebauten Orte, die der Einhegung des Kinderspiels im städtischen öffentlichen Raum dienen, das Spiel fördern und zu „Territorien der Kinder“ (Moore 2019 [1986]) werden können.

Das Spiel zeichnet sich vor allem durch seinen freien und freiwilligen, kreativen und fundamental anti-utilitaristischen Charakter aus, ebenso wiedurch die Ungewissheit seines Ausgangs (Caillois 2012 [1958]; Huizinga 1951).
„Wir spielen nur, wenn wir wollen, wann wir wollen und so lange wir wollen. In diesem Sinne ist das Spiel eine freie Tätigkeit. Es ist auch eine ungewisse Tätigkeit. Der Zweifel über den Ausgang muss bis zum Schluss bestehen bleiben. [...] Das Spiel besteht in der Notwendigkeit, eine Antwort zu finden und unmittelbar zu erfinden, die innerhalb der Grenzen der Regeln frei ist. Dieser Spielraum des Spielers, dieser seinem Handeln eingeräumte Freiraum, ist wesentlich für das Spiel und erklärt zum Teil das Vergnügen, das es hervorruft. " (Caillois 2012 [1958]: 39 f.; Übers. d. A.)

In diesem Sinne ist das Spiel, wie Benjamin feststellt, in erster Linie eine freie und kreative Beziehung zu Objekten, Materialien und Spuren (Tarragoni 2015) und hat einen grundsätzlich transgressiven Charakter.

Die Charakteristika des städtischen Raums und der geographischen Kontexte von Kindern im Allgemeinen sowie ihre sozialen und kulturellen Rahmenbedingungen sind von zentraler Bedeutung für das Verständnis des kindlichen Spiels und des Verhältnisses von Kindern zu Spielplätzen (Valentine/ McKendrick 1997). In Europaund den Vereinigten Staaten wurde dieses Thema relativ ausführlich untersucht, ebenso wiedieGeographien der Kindheit im Allgemeinen (Ansell 2019; Hanson etal. 2018; Holloway 2014). Für Mexiko-Stadt beschränkt sich diebisherigeForschungauf dieBeziehung besonders marginalisierter Gruppen von Kindern (insbesondere auf der Straße lebende oder arbeitende Kinder) zum städtischen Raum (Pérez López 2016).

Die Einbeziehung weiterer Gruppen von Kindern sowie des Themas Spielplatz fand in dieser Forschung nur sehr marginal statt (Gülgönen/ Laboratorio para la ciudad 2016). Die wenigen vorhandenen Studien in diesem Kontext zeigen, dass europäische und nordamerikanische Spielplätze stets Modellcharakter für Spielplätze in Mexiko hatten (Solano Rojas 2018). Anhand eines kurzen historischen Überblicks, der sich auf dieseStudien bezieht, sowie einer Untersuchungzeitgenössischer Spielplätze werde ich im Folgenden erläutern, wie einerseits soziale, historische und kulturelle Repräsentationen von Kindheit und andererseits städtische 
Transformationsprozesse die Form von Spielplätzen beeinflusst haben. Die Diskussion der jüngeren Geschichte von Spielplätzen in Mexiko, ihrer aktuellen Form und ihrer weltweiten Homogenisierung fußt auf meiner Forschungzu diesem Themain Mexiko-Stadt, insbesondere auf zahlreichen Interviews mit Beamt innen und Stadtplaner innen.[1] Auf diese Weise möchte ich beleuchten, wie diese Räume dazu beitragen, ein globales Bild von Kindheit zu prägen. Dabei geht es mir nicht darum, von einer „globalen Kindheit" als solcher zu sprechen, sondern zu untersuchen, wie der globale Kapitalismus Kindheiten in unterschiedlichen Kontexten maßgeblich beeinflusst (Hanson et al. 2018) und wie Spielplätze die Verbreitung eines normativen Bildes von Kindheit - von den USA und Europa ausgehend - in anderen Weltregionen vorantreiben. Gleichzeitig möchte ich zu einer Reflexion über die sich verändernde Natur des Spielens (Murnaghan 2019) beitragen und fragen, ob und inwiefern sich die Transformation des Spielplatzes darauf auswirkt, wie Kinder spielen.

\section{Die Erfindung des Spielplatzes: Ausschluss und Moral}

Dievon Pieter Bruegel dem Älteren im 16. Jahrhundert in Die Kinderspiele und Die J äger im Schnee gemalte Stadt-als-Spielpatz, in der sich Kinder jeden Alters und aller sozialen Milieus frei im städtischen Raum bewegen, ist in Europa einem ,langen Prozess der Privatisierung“ von Kindheit gewichen. In dessen Verlauf wurden Kinder allmählich von den Straßen verbannt. Gleichzeitig wurden spezifische Gelände für sie geschaffen, in denen sie all jene Aktivitäten ausüben sollen, die ihnen körperlich, sozial und moralisch „guttun“: draußen sein und spielen (Ariès 1993).

Die Verbannung der Kinder von der Straße sollte sie vor den Lastern der Stadt schützen, aber gleichzeitig auch die Stadt vor einer eventuell unerwünschten Präsenz bewahren, die sich nicht an die allgemein akzeptierten Regeln hält (Hart 2002). So wurden Spielplätze Ende des 19. J ahrhunderts in den Vereinigten Staaten und in England von philanthropischen Vereinen geschaffen - nicht um Kinder zum Spielen zu animieren, sondern aus hygienischen und moralischen Motiven. Kindern sollten adäquate Räume für ihre körperliche und geistige Entwicklung geboten werden. Im Gegensatz dazu stand das vorherrschende Bild der Straße als Ort der Verderbtheit. Zugleich wollte man sowohl die Räume als auch die (Frei-)Zeit der Kinder, insbesondere der Arbeiterkinder, kontrollieren (Hart 1986). WieFranklin D. Roosevelt es 1907 in seiner Zeit als NewYorker Polizeipräsident ausdrückte: „City streets are unsatisfactory playgrounds. "(Cazalis 2018: 184)

In Mexiko entstanden die ersten Spielplätze zu Beginn des 20. J ahrhunderts, wobei die Form europäischer Kinderspielplätze importiert wurde. Der Einfluss des Entwicklungsmodells großer nordamerikanischer und europäischer Städte auf die Umgestaltung des Stadtgefüges ist dabei deutlich zu erkennen. So geht der Bau von Spielplätzen mit dem politischen Willen zur „Modernisierung der Stadt“ einher und ist untrennbar mit der Stärkung sozialer Institutionen und der Schaffung kollektiver Wohnungen, Krankenhäuser und Erholungsräume verbunden (Solano Rojas 2018).

Im 20. Jahrhundert veränderte diezunehmende Präsenz des Automobils dieGestalt der Städte - dies wird oft als Hauptursachefür das Verschwinden 
der Kinder von den Straßen und die Vervielfachung der Enklaven für kindliches Spiel genannt (Borja 2010; Lynch 1977; Wridt 2004). DieStraßen sind nicht länger „gemeinschaftliche“ Räume, „place[s] of popular sociality, [...] play space[s] for kids“, sondern werden zum „public space dominated by the advent of the automobile" (Harvey 2012: 117). Die Vervielfachung der Spielplätze ist dank massiver staatlicher Investitionen möglich; staatlich angestellte erwachsene Aufsichtspersonen überwachen das kindliche Spiel.

Die aufkommende Kritik an dieser dem Auto unterworfenen Stadt ist eng verbunden mit der Kritik am funktionalistischen Urbanismus. Dieser trägt dazu bei, die Nutzungsvielfalt von Räumen einzuschränken, das Verschwinden gemeinsam genutzter Räume voranzutreiben und gleichzeitig die Schaffung spezifischer Orte für Kinder notwendig zu machen. Auch das Leben auf den Gehwegen nimmt dadurch ab, die „Augen der Straße“ - die eine natürliche, anonyme Aufsicht der Kinder im öffentlichen Raum ermöglichen - verschwinden (J acobs 1992 [1961]).

Seit ihrer Entstehung ist einer der Hauptkritikpunkte an Spielplätzen folglich, dass sie Kinder vom städtischen Raum absondern. Mitihrem spezifischen (und oft identischen) Mobiliar - Rutschen, Klettergerüste, Schaukeln etc. - können sie nicht die Nutzungsvielfalt und Begegnungsmöglichkeiten ersetzen, diedie Straßezu einem grundsätzlich spielerischen Raum machen. VieleAutor_innenderzweiten Hälftedes20.J ahrhunderts betrachteten Spielplätze deshalb als zutiefst langweilige Räume, die die Spielbedürfnisse von Kindern nicht befriedigen können (J acobs 1992 [1961]; Ward 1990 [1978]).

\section{Der Spielplatz als kreativer Raum}

In Europa kam es in der Nachkriegszeit zu einem Umdenken hinsichtlich der Spielplätze, während gleichzeitig der Staat verstärkt in die Stadt investierte. Amsterdam ist in diesem Sinne emblematisch: Während auf den durch Bombenangriffe entstandenen Brachflächen Spielplätze entstehen, versucht der Architekt Aldo van Eyck, Kinder „in das Alltagsgefüge der Stadt“ zu integrieren (van Eyck 2014 [1962]). Entgegen der früheren Ausgrenzungsdynamik steht hier der Wille zur Einbeziehung von Kindern „als wesentliche[m] Bestandteil der Stadt" im Mittelpunkt (ebd.: 127). So sind Spielplätze nicht umzäunt und als „Räume für alle“ gedacht. Gestaltung und Standorte der Spielgeräte werden als wesentlich dafür erachtet, dass Kinder sie sich durch Erkundung und Spiel zu eigen machen können (Moore 2019 [1986]). Siebestehen aus einfachen geometrischen Elementen und sind so konzipiert, dass Kinder frei in und mit ihnen spielen können. DieWahl vielfältiger, aber einfacher Formen und Materialien deckt sich mit Walter Benjamins Kritikan der Spielzeugindustrieder 1920erJ ahre, diedem Wesen des Spiels widerspreche:

„Heute darf man vielleicht schon hoffen, den gründlichen Irrtum zu überwinden, der da vermeint, der Vorstellungsgehalt seines Spielzeugs bestimmedas Spiel des Kindes, da es in Wahrheit eher sich umgekehrt verhält. Das Kind will etwas ziehen und wird Pferd, will mit Sand spielen und wird Bäcker, will sich verstecken und wird Räuber oder Gendarm. [...] Denn je ansprechender im gewöhnlichen Sinne Spielsachen sind, 
umso weiter sind sie vom Spielgeräte entfernt; je schrankenloser in ihnen die Nachahmung sich bekundet, desto weiter führen sie vom lebendigen Spielen ab. [.. .] Nachahmung- so lässt sich das formulieren - ist im Spiel, nicht im Spielzeug zu Hause.“ (Benjamin 1969: 65)

Dieses Anliegen findet sich in den Entwürfen zahlreicher europäischer und nordamerikanischer (Borja-Villel etal. 2014; Burkhalter 2018; Cazalis 2018) sowiemexikanischer (Solano Rojas 2018) Designer_innen und Architekt_innen der 1950er bis 1970erJ ahre wieder, deren Ideen radikal mit den moralisierenden Vorstellungen der Gründer_innen der ersten Spielplätzebrechen.

Im gleichen Zeitraum erfolgen in Mexiko große staatliche Investitionen in die Modernisierung der Städte - die Erneuerung der öffentlichen Infrastruktur und den Ausbau der Erholungsgebiete in den Großstädten. Parks und Kindergärten werden im ganzen Land saniert und neu angelegt. Dafür sind auch hier starke öffentliche Institutionen entscheidend, etwa die Ende der 1950erJ ahre entstehenden Centros Deportivos Ferrocarrileros (Eisenbahn-Sportzentren) oder das Nationale Institut zum Schutze der Kindheit (Instituto Nacional de Protección a la Infancia, INPI), das 1961 gegründet wird. Der Neubau großer Wohnkomplexeund-siedlungen stellt einen weiteren Impuls für die Schaffung von Spielplätzen dar, unter anderem in MexikoStadt (Solano Rojas 2018).

Bei der Gestaltung der Spielplätze ist eine ähnliche Kreativität zu beobachten wie zur gleichen Zeit in Europa und den Vereinigten Staaten. Das zwölf Meter hohe raketenförmige Spielgerät mit drei Riesenrutschen, das 1964 im größten dieser Wohnkomplexe, dem Complejo Urbano NonoalcoTlatelolco,[2] aufgestellt wird, ist aufgrund seiner schwindelerregenden Höhe sinnbildlich hierfür. Mitte der 1970erJ ahre wird es allerdings wieder entfernt, nachdem es zu tödlichen Stürzen kam.

Der ästhetische Anspruch fällt hier zusammen mit dem pädagogischen Interesse, Kreativität und Risiko als grundlegende Elemente des kindlichen Spiels zu fördern. Es ist einerseits die Möglichkeit des freien und vielfältigen Spiels, diees Kindern erlaubt, sich den Spielplatz zu eigen zu machen. Andererseitsist dieräumlicheAnordnung des SpielplatzesinnerhalbeinesUmkreises, in dem sich die Kinder autonom bewegen können, ausschlaggebend. So wird der Spielplatz zu einer räumlichen Referenz für Kinder und zu einem Ort, an dem sie eine gewisse Freiheit erleben können (Moore 2019 [1986]).

Als radikalster Vorschlag in dieser Richtung gilt der Abenteuerspielplatz, der Mittedes 20. J ahrhunderts in mehreren Städten Europas und - in geringerem Maße - in den Vereinigten Staaten populär wird.[3] Ohnevorgefertigte Spielgeräte stellt er Kindern die Werkzeuge und die Freiheit zur Verfügung, die Räume, die sie sich wünschen, zu bauen und permanent umzugestalten. Abenteuerspielplätze fungieren als Lücken im städtischen Raum, in denen Kinder forschen, bauen, Risiken eingehen und bis zu einem gewissen Grad die Freiheit wiedererlangen können, die sie einst aufden Straßen und Brachflächen der Stadt hatten - wenn auch unter der Aufsicht von Erwachsenen, die die Kinder hierbei unterstützen (und nicht etwa überwachen).

Diese Räume basieren auf einer nicht-autoritären, kollaborativen Pädagogik, weshalb anarchistische Theoretiker_innen wie Colin Ward sie als „a free society in miniature" bezeichnet haben (Ward 1961: 193). Sie stellen 
eineantiautoritäreAntwort auf das Problem der Des-Integration von Kindern in das städtische Leben dar:

„The authoritarian solution to this need is to providean area of tarmac and some pieces of expensive ironmongery in the form of swings, seesaws and roundabouts, which providea certain amount of fun (though because of their inflexibility children soon tire of them), but which call for no imaginative or constructive effort on the child's part and cannot be incorporated in any self-chosen activity." (Ward 1961: 193 f.)

Spielplätze offenbaren eine bestimmte Vorstellung von Kindheit und, allgemeiner, ein bestimmtes Gesellschaftsmodell. Ab den 1980er J ahren verstärken sich Phänomene der Überbehütungvon Kindern und einer damit einhergehenden Überwachung. Dies ist sicherlich einer der Hauptgründe, warum die bei Kindern sehr beliebten Abenteuerspielplätze an Bedeutung verlieren und stattdessen Sicherheitsstandards dieGestaltung der Spielplätze bestimmen. So tragen einerseits dieden Abenteuerspielplätzen innewohnende Gefahr, andererseits ihre Kosten (sie erforderten dieAnwesenheit bezahlter Aufsichtspersonen) zu ihrem Niedergang bei.

\section{Versicherheitlichung und Überwachung: Desinvestition und Verödung des öffentlichen Raums}

Ab Ende der 1970er J ahre markiert der Rückzug des Staates aus der Finanzierung und Erhaltung von Spielplätzen den Beginn eines neuen Kapitels in deren Geschichte. Das gilt für Mexiko-Stadt ebenso wie für viele europäischeund nordamerikanische Städte. In NewYorkführen dieausbleibende Instandsetzung von Spielplätzen und die Streichung des Budgets für die Bezahlung erwachsener Aufsichtspersonen zu ihrem Verfall und zur Besetzung durch andere, als unerwünscht angesehene Gruppen, etwaJ ugendliche aus ärmeren Schichten. Hier, wiein Mexiko-Stadt, bleiben Kinder aus wohlhabenden Familien daraufhin den Spielplätzen fern und nutzen verstärkt private Räume.

In Mexiko-Stadtist die - gefühlt oder real - zunehmendeUnsicherheit im öffentlichen Raum (nicht mehr nur auf der Straße) einer der Hauptfaktoren, die zum Fernbleiben der Kinder von Spielplätzen und anderen öffentlichen Plätzen beitragen. Die realeZunahmevon Gewaltdelikten, ihre Dramatisierung in den Massenmedien und ihre Instrumentalisierung durch die Regierungen befördert ein allgegenwärtiges Gefühl der Unsicherheit (Guerrien 2001; Pansters/Berthier 2007). Gewaltistzu einem „,Gespenst' geworden, das täglich und ungehindert durch lateinamerikanische Metropolen streift" (Pansters/Berthier 2007: 508) und das dieArt und Weiseverändert, auf die Stadt und insbesondere der öffentliche Raum erlebt wird. In lateinamerikanischen Städten führt dies ab den späten 1980er J ahren zu einer radikalen Wende in der Produktion der Stadt (Caldeira 2007; Capron 2012): Zum Schutz vor Kriminalität werden hohe Mauern um dieHäuser der Mittelund Oberschicht errichtet. Sie zeugen von dem Wunsch, sich vom Rest der Stadt abzusondern, von einer wachsenden Distanz zum öffentlichen Raum und zu Menschen aus anderen sozialen Schichten. „Die Begegnungen im öffentlichen Raum werden jeden Tag angespannter, sogar gewalttätiger, 
denn ihre Referenz sind die Stereotypeund Ängsteder Menschen. Spannung, Trennung, Diskriminierungund Misstrauen sind dieneuen Kennzeichen des öffentlichen Lebens.“(Caldeira 2007: 363)

Dieses Gefühl der Unsicherheit wirkt sich auch darauf aus, wieKinder mit der Stadt in Beziehung treten. Über reale Risiken - etwa im Straßenverkehr - hinaus steigt die Angst vor Entführungen stark an. Sie hält Eltern davon ab, ihre Kinder im öffentlichen Raum allein zu lassen und hat deren ständige Überwachung zur Folge. Diese Angst verinnerlichen auch Kinder (Gülgönen/ Corona 2015). Aufgrund des Fehlens verlässlicher Statistiken über Kindesentführungen in Mexiko kennen wir das tatsächliche Ausmaß dieses Phänomens nicht. Interessant ist, dass dieFigur des robachicos (Kinderräubers) schon lange vor der Verschlechterung der Sicherheitslage auftauchte und dass Medien schon ab den 1950erJ ahren ähnliche Narrativeaufnahmen und damit Ängste in der Stadtbevölkerung schürten (Sosenski 2018: 105).

In ständiger Begleitung eines Erwachsenen (ausschließlich mit dem Auto) von einem privaten Raum zum anderen transportiert, erleben Mittel- und Oberschichtskinder die Stadt wieeinen Archipel. Diese „Insularisierung“ der Stadterfahrung charakterisiert das Leben von Stadtkindern in sehr unterschiedlichen städtischen Kontexten und unabhängig vom realen Grad der Unsicherheit (Gillis 2018; Karsten/van Vliet 2006). Insbesonderein Mexikos Städten sind diese Inseln vor allem Orte des Konsums, zu denen Kinder ihre Mütter oder Väter begleiten (Gülgönen 2021) - was die Ideeder Stadt selbst obsolet macht (Giglia 2003).

Auch die Spielplätze sind solche Inseln. In Mexiko-Stadt ist der Spielplatzbesuch in erster Linie ein gelegentlich stattfindendes Familienerlebnis. Dort stehen Kinder unter der ständigen Aufsicht von Erwachsenen, es sind

Abb. 1 Delegación Miguel Hidalgo, Mexiko-Stadt, 2016 (Quelle: Börries Nehe) keine Orte, an denen Kinder andere Kinder treffen oder kennenlernen (Gülgönen/ Corona 2019). Somit können diese Plätze auch nicht zu ihren Territorien werden, zu räumlichen wiesozialen Bezugspunkten (Lehman-Frisch/ Authier/ Dufaux 2012; Moore 2019 [1986]).

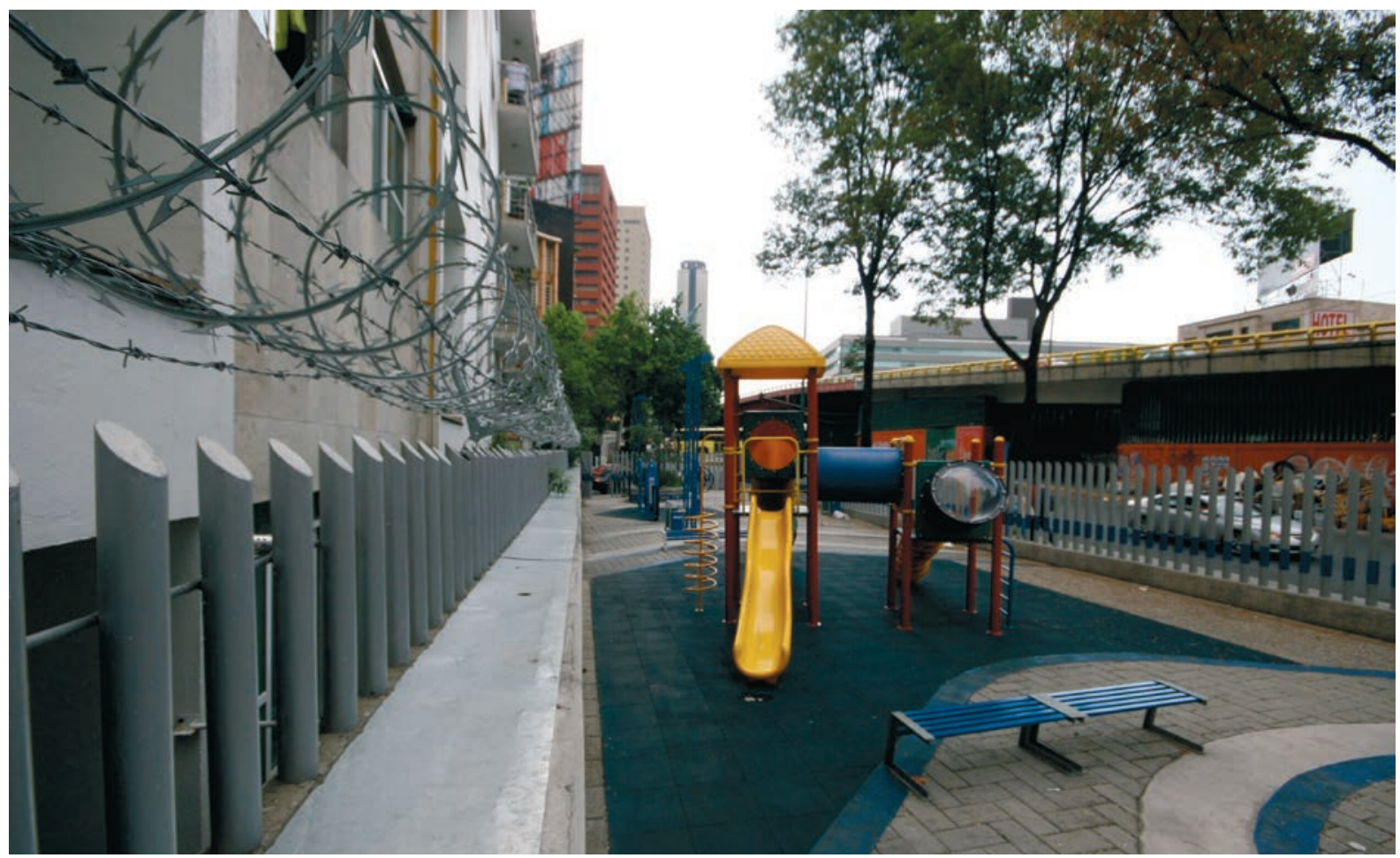


Dass Überwachung und Kontrolleimmer mehr zu zentralen Aspekten der Kinderbetreuung werden - auf Kosten des freien Spiels und der kindlichen Autonomie - , ist nichtnurin Lateinamerika zu beobachten. In derwestlichen Literatur über Kindheit und Stadt der letzten 30 J ahre bilden Ängste und der damit verbundene Wille zur permanenten Überwachung eine Konstante im Verhältnis von Kindern und ihren Eltern zur Stadt (Carver et al. 2013; Hillman/Adams/Whitelegg 1993; Kyttä etal. 2015; Rissotto/ Giuliani 2006; Tyagi/ Raheja 2020). Damit korrespondiert ein weltweit vorherrschendes Bild von Kindheit „as an idyllic, happy time when a child is temporally set apart from the adult world (although there are multiple and conflicting definitions of the age at which this division occurs) and is free from the responsibilities of adulthood" (Valentine/ McKendrick 1997). Gleichwohl werden Kinder aus nichtbürgerlichen Milieus per se von diesem Bild ausgeschlossen und im Gegensatz dazu als Bedrohung wahrgenommen (Rodrigues Breitman 1994).

\section{Architektur der Angst: Spielplätze ohne Spiel}

Die Versicherheitlichung der Stadt hat einen direkten Einfluss auf die Architektur von Spielplätzen. Hier materialisiert sich die „Hyperüberwachung der Kinder“" (Katz 2006) in Form eines „paranoiden Versuchs, risikolose Umgebungen zu schaffen" (Hart 2002: 144): umzäunte Räume ohne Hindernisse, die die Sicht versperren könnten; Spielgeräte, die so konzipiert sind, dass sie das Verhalten der Kinder antizipieren und nicht zulassen, dass sie fallen oder sich verletzen; stoßgedämpfte Böden und Überwachungskameras. Das Design der Spielplätze ist nicht mehr vom Wunsch bestimmt, freies kindliches Spiel zu ermöglichen, sondern es soll damit verbundene Risiken bestmöglich reduzieren.

Die Priorisierung der Sicherheit hat in den vergangenen J ahrzehnten zu einer neuen Präsenz von Staatlichkeit auf Spielplätzen geführt, und zwar in Form von Reglementierungen. Dieersten Vorschriften für den Bau von Spielplätzen gab es in den 1970er J ahren in den USA und einigen europäischen Ländern. Insbesondere in den Vereinigten Staaten führten Klagen gegen städtische Gemeinden aufgrund von Unfällen auf Spielplätzen zur Schaffung strikter Vorschriften und Sicherheitsstandards. Auf europäischer Ebene wurden Ende der 1990erJ ahreverbindliche Normen für die Gestaltung und Instandhaltung von Spielplätzen eingeführt (J ansson 2009).[4]

Befördert durch das Wachstum der globalen Spielzeugindustrie verbreiteten sich europäische und US-amerikanische Normen in den vergangenen J ahren auch in anderen Teilen der Welt. Zudem führte der Import von in den USA oder Europa produzierten Spielgeräten (und später dieEntwicklung lokaler, mitdenselben Standards arbeitenden Industrien) dazu, dass weltweit vollkommen identische Spielplätze entstanden: dieselben Spielgeräte, dieselben grell leuchtenden Farben, dieselben stoßgedämpften Böden.

In Mexiko-Stadt existieren keine klaren Richtlinien für Spielplätze, abgesehen von einigen Dokumenten, die nicht groß im Umlauf sind. In den meisten Fällen sind Gebietskörperschaften für die Spielplatzflächen zuständig und der Gestaltungsprozess wird direkt an - meist ausländische Unternehmen vergeben, die Spielgeräte verkaufen: 
„Im Grunde genommen, die Spielgeräte [...] ich zumindest habe viele davon aus dem Katalog gekauft. Ich war für [den Bezirk] Gustavo I. Madero verantwortlich, und wir haben viele Spielplätze gebaut. Also ich habe sie dem damaligen Bezirksbürgermeister präsentiert: „Dies hier mag ich', und er sagte mir: ,Nein, ich mag diese Farbelieber. ' Das waren schöne Kataloge, amerikanische, ich weißnicht, alle Spielgeräte waren amerikanisch. [...] Alle Spielgeräte, die ich aufgestellt habe, kamen aus dem Ausland. Es gibt auch mexikanische [Firmen] [...]. Was ich sagen will, ist, dass der Großteil, sagen wir mal 80 Prozent, außerhalb Mexikos produziert wird. [...] Aber es gibt Unternehmen, die in Mexiko eine gesetzliche Vertretung haben. Die Tatsache, dass [dieGeräte] in einem anderen Land hergestellt werden, bedeutet nicht, dass die Mexikaner nichts zu sagen haben. “[5]

Der Wunsch, europäische oder US-amerikanische Spielplätze zu kopieren, sowiedieMarktdominanz der per Katalog gekauften Spielgeräte haben dazu geführt, dass viele Sicherheitsvorschriften ebenfalls importiert wurden, ohne den lokalen Kontext zu berücksichtigen (Gülgönen/ Laboratorio para la ciudad 2016). Dabei werden Spielgerätehersteller_innen oft als „Experten“ für das Spielen angesehen:

\begin{abstract}
„Siesind dieExperten fürs Aufstellen von Spielgeräten, siekönnen uns jenes Modul empfehlen, das mit der Schaukel, mitder Rutsche, mitdem Spinnennetz, mit dem Handlauf [...]. Also was die Sicherheit angeht, das liegt bei den Lieferanten. Können sie etwas empfehlen? Sicherlich, denn der Lieferant will Ihnen natürlich den kompletten Bausatz verkaufen, und er wird Ihnen sagen: ,Kauf auch den Gummiboden.' Der funktioniert aber auch wirklich. [...] Die Unternehmen haben diesen Projektbereich. Wenn Sieein Spielplatzgerätinstallieren wollen, gehen Sie [...] zu der Firma und sagen: ,Ich habe 100 Quadratmeter und ich möchte Spielplatzgeräte installieren, welche empfehlen Sie?‘ Und siemachen dir ein Projekt und siearrangieren die Spielgerätefür dich, sie platzieren sie so, dass sie nicht in der Nähe eines Astes sind. Und der Boden, den sie angepriesen haben, der ist aus Gummi, um keine Unfälle zu verursachen. Als man damit anfing, diese Spielplätze zu bauen, war das Konzept, dass man siewie einen Laufstall gestaltete, um die Kinder ein wenig mehr unter Aufsicht zu halten. “[6]
\end{abstract}

Mit dem zentralen Sicherheitsargument der Stadtverwaltung werden die alten Spielgeräte aus Holz und Metall ausgetauscht, was oft mit erheblichen Investitionen verbunden ist (vgl. Abb. 2). Oft stellt dann die ausbleibende Instandhaltung der Geräte ein Problem dar. Das geschieht gegen die Empfehlungen lokaler Instanzen, „,witterungsbeständige, leicht zu wartende und zu reparierende Materialien wie Eisenrohre, Zement und Holz“ zu nutzen (SEDESOL 2007).[7]

Als weiteres Argument für die Anschaffung der neuartigen Spielgeräte fungiert ihr Design, das die Entwicklung der Kinder stimulieren soll:

„Andere Arten von Spielen, wie Spinnennetze, die ein bisschen mehr kosten und die ihnen helfen, ein bisschen mehr Geschicklichkeit, Fähigkeiten zu entwickeln. [...] Die Idee ist jetzt, die Spielgeräte mit 


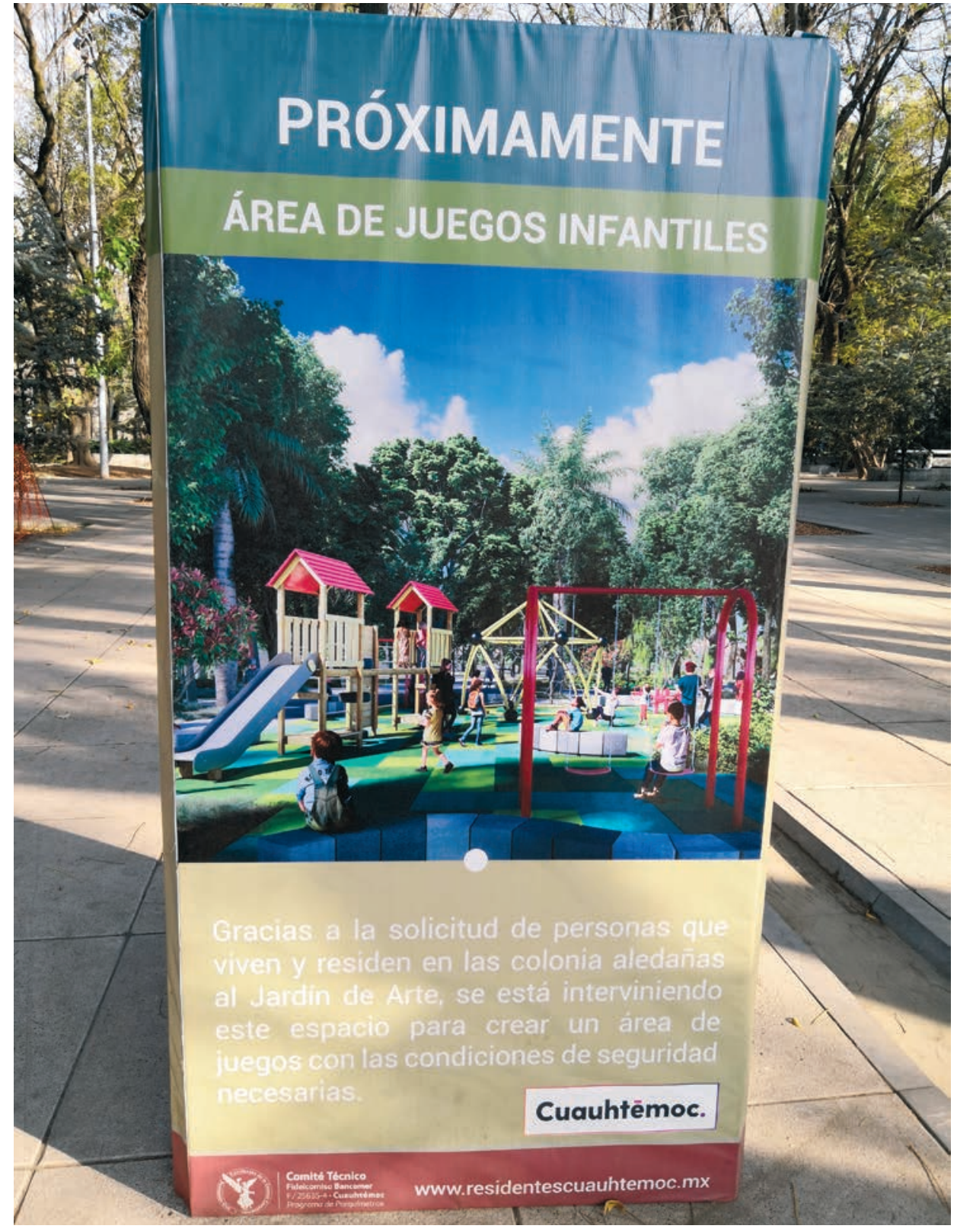

dieser Art zu ersetzen, die die Kinder stimulieren, sodass sie auf eine andere Art und Weise spielen können, wo siereingehen, hochklettern und runtergehen können. Vielleicht kostet sie das ein bisschen mehr Arbeit, aber es hilft ihnen, ein bisschen mehr darüber nachzudenken, wie sie es anstellen sollen. [...] Ich meine, es gibt verschiedene Altersgruppen, von drei bis sieben J ahren, die erste Etappe, da haben alle Rutschen unterschiedliche Texturen, damit man dies... also, um Dingsda anzuregen... das motorische System oder ich weiß nicht was. Also diese Geräte haben irgendwas Besonderes. “[8]

Diese Spielgerätesind bereits in vielen Städten weltweit, in etlichen Einkaufszentren und Fast-Food-Restaurants aufgestellt worden, stets mit den gleichen Argumenten: Sicherheit und Stimulation der motorischen Fähigkeiten von Kindern. Sie implizieren eine globale Kindheit und entsprechen einer Kommerzialisierung der familiären Freizeit. Denn sieerlauben es den Eltern oder Begleitpersonen, ihre Aufmerksamkeit anderweitig (auf den Konsum) auszurichten, ohne sich Sorgen um die Sicherheit ihrer Kinder machen zu müssen (McKendrick/ Bradford/ Fielder 2001).
Abb. 2 Jardín del Arte, Mexiko-Stadt, 2021. Übersetzung des Aufdrucks: „Kinderspielplatz in Vorbereitung. Auf Wunsch von Menschen, die in der Nachbar_innenschaft des Jardín del Arte [Kunstgarten] leben und wohnen, wird dieser Raum umgestaltet und ein Spielplatz mit den notwendigen Sicherheitsvorkehrungen geschaffen." (Quelle: Óscar Sánchez Hernández) 
Abb. 3 CuitlahuacPark, Delegación Iztapalapa, MexikoStadt, 2016 (Quelle: Börries Nehe)
Indem siejedoch ausschließlich Sicherheitsaspekten entsprechen, negieren solche Geräte das eigentliche Wesen des Spiels - das Eingehen von Risiken - und seinen freien, erkundenden Charakter. Ein Design, das explizit darauf ausgerichtet ist, das Verhalten von Kindern zu antizipieren und Risiken so weit wie möglich zu minimieren (Treppensteigen, eine Plastikröhre durchrutschen), ist damit unvereinbar (Hart 2002). Damit stehen diese Spielgeräte in einem starken Widerspruch zu den Entwürfen spielerischkreativer Räume, dieich im vorigen Abschnitt dargestellt habe. Das Problem kann wohl kaum darin bestehen, dass Designer_innen unterschiedliche Auffassungen von der Natur des kindlichen Spiels haben. Stattdessen erscheint es mir vielversprechend, das globale Geschäft mit Spielgeräten und Spielplätzen und die damiteinhergehende Kommerzialisierung der Stadt genauer zu untersuchen.

\section{Die Rolle von Spielplätzen in der neoliberalen Stadt}

Oft investieren Stadtverwaltungen in teure Spielplatzgeräte, dievon Kindern gar nicht genutzt werden, was häufig ihrer ungünstigen Lage geschuldet ist:

„[...] zwischen 2007 und 2010 gab es hier im Bezirk ein interessantes Programm zur Wiederherstellung öffentlicher Räume durch den Bau von Spielplätzen. Viele von denen, die Sie im Bezirk sehen, stammen aus dieser Zeit. Einige hatten keinen Erfolg, andere hatten Erfolgund sind bis heute sehr gut gepflegt. Ich weiß nicht, ob Sie den einen [Spielplatz] kennen, der hier auf der [Stadtautobahn] Periférico fast auf der Höhe des Museums für Militärkartografie ist, Ecke Periférico und Observatorio. Es ist wahr, dass die Lage sehr schlecht ist. Es gibt dort kein hohes Fußgängeraufkommen, und wenn es Menschen gibt, steigen sie von einem Bus in den anderen um, Leute spazieren dort nicht und kommen auch nicht aus einer Schule, da gibt es keine Anbindung für eine Mutter, die mit ihren Kindern spazieren geht. Ich denke, das ist der Grund, warum er nicht erfolgreich war. "[9]

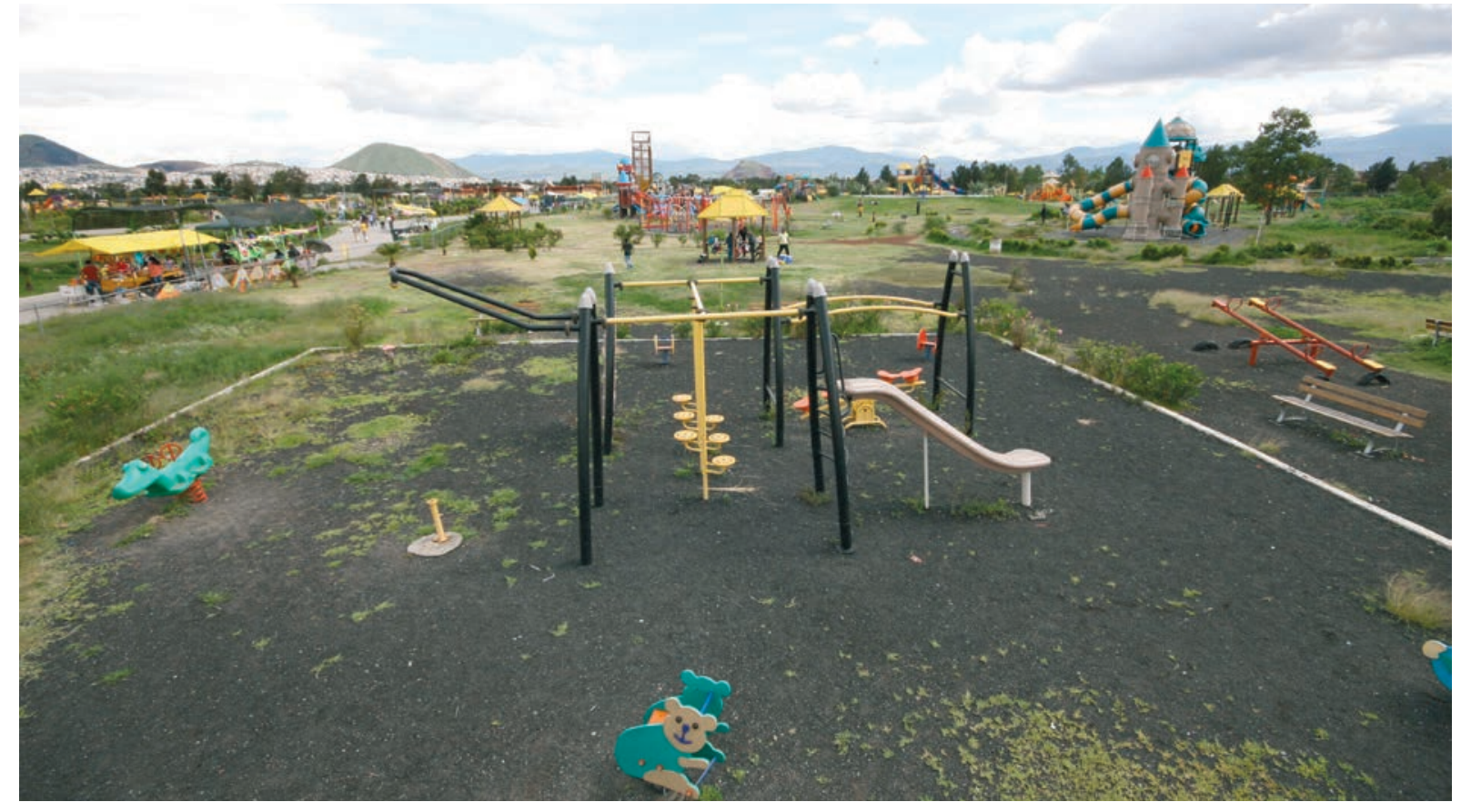


„Im Cuitlahuac-Parkhat dievorherigeVerwaltung Spielefür Kindermit Behinderungen aufgestellt. Und sie sind sehr schön, sehr modern, sie haben etwa eine Million Pesos gekostet. Aber ich denke, es fehlt uns an Publicity, der Park ist sehrverlassen. In der letzten Regierungsperiode wurden dort viele Ressourcen investiert, aber ich sehe nie, dass die Leute in den Park gehen würden... Ich sehe nie, dass Kinder dorthin gehen, niemand bringt sie dorthin. “[10]

Mit den sogenannten pocket parks und bajopuentes (wörtlich: UnterBrücken) hat man versucht, ein quantitatives Ziel zu erreichen: einen Zugang zu Erholungsräumen für möglichst viele Menschen. Dafür wird die Stadt auf den wenigen noch verbliebenen Freiflächen mit Spielgeräten gefüllt. Pocket parks greifen in Mexiko-Stadt in kleineren Ausmaßen ein Modell auf, das weltweit in zahlreichen Städten umgesetzt wird.[11] Als bajopuentes werden Flächen bezeichnet, die sich unter Autobahnen befinden und von privaten Unternehmen mit Spielgeräten bestückt werden. Dies setzt die Praxis der „linearen Parks“ fort, die sich oftmals auf Mittelstreifen zwischen viel befahrenen Straßen und unter Hochspannungsleitungen befinden, was der Bekämpfung von Unsicherheitsgefühlen im städtischen Raum dienen soll (SEDESOL 2007: 42). Die Verortungvon Spielplätzen in diesen prekären Räumen zeugt von der marginalen Position, die Kindheit innerhalb der Stadtlandschaft zugesprochen wird.

Es stellt sich die Frage, woher der Wille rührt, so viele dieser Orte in der Stadt einzurichten. DieAntwort könntein den Verbindungen und Interessenkonflikten zwischen Unternehmen und Beamt_innen zu finden sein. Dafür spricht der Fall eines Interviewpartners, der zunächst für ein Unternehmen arbeitete, das mit dem Bau von Spielplätzen beauftragt war, und dann als Beamter in jener Behörde arbeitete, die für die Ausschreibungen der Spielplätze zuständig war:

Abb. 4 Bajopuente, Mexiko-Stadt, 2016 (Quelle: Börries Nehe)

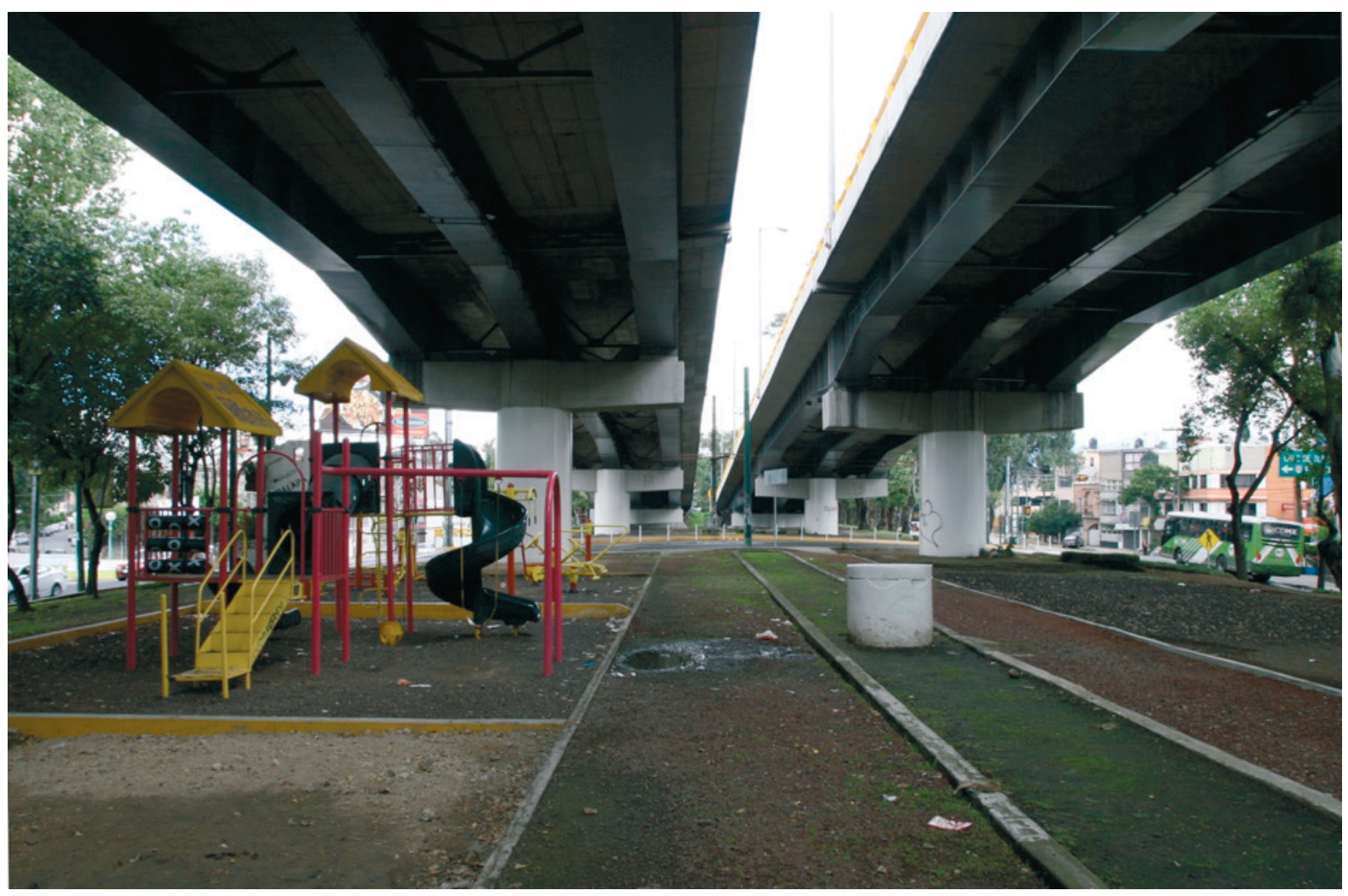


„Kennen Sie[die Ringautobahn] Rio Churubusco? Dort, wo die Spielgeräte aufgestellt wurden? Das habe ich vor sieben J ahren entworfen. Ich warmit einer Firma dabei, wir haben dieAusschreibung gewonnen [...] Wir haben den Wettbewerb 2012 gewonnen, für das Design. Wir! Wir wurden von der Stadtregierung beauftragt, von irgendeinem Bezirk, mit Budget des Bundes. [...] Für diese Marke, diese Firma habe ich gearbeitet, aus Alabama, wo diese Spielgeräte hergestellt werden, und wir haben die Ausschreibung gewonnen [...]. Damals war ich kein Beamter, ich war im Unternehmen. Wir als Unternehmen, ich meine, ich war zu dieser Zeit nicht im öffentlichen Dienst, wir haben all diese Arbeit gemacht.“[12]

Von Beginn an ist die Einrichtung von Spielplätzen mit dem Bewusstsein verbunden, dass sie zurWertsteigerung nahegelegener Immobilienprojekte beitragen: Befindet sich ein Spielplatz in der Nähe, sind wohlhabendere Familien eher geneigt, ein Haus zu kaufen. In New York betreibt die Immobilienbranche seit den 1920er J ahren Lobbyarbeit, um über Public-privatePartnerships den Bau von Spielplätzen zu fördern. In einer 1928 erschienenen Ausgabe der Zeitschrift der Playground Association of America (PAA) ist zulesen:

„It is a proved fact that beautiful play areas not only enhance real estate values in the surrounding neighborhood, but also counteract the discordant emotions of the children using them, overcoming the usual destructivetendencies of children who are served by unattractive, barren play spaces. "(Zit. nach Cazalis 2018: 182)

In dieselbe Richtung zielt die Instrumentalisierung des Begriffs „öffentlicher Raum“ in der neoliberalen Stadt: Seine breite Verwendung durch Designer_innen, Architekt_innen, Stadtplaner_innen und Manager_innen in den 1980er und 1990er J ahren in Europa und den USA korrespondiert mit strategischen staatlichen Investitionen im öffentlichen Raum am Ende des 20. und zu Beginn des 21. J ahrhunderts (Delgado 2015; Harvey 2012).

In Mexiko-Stadt wurde 2008 die Behörde für den öffentlichen Raum (Autoridad del Espacio Público, AEP) gegründet, diedortvorallemstrategische Projekte planteund durchführte. Neben den schon erwähnten pocket parks und bajopuentes war die AEP etwa für den Bau zahlreicher Fußgängerzonen im historischen Zentrum und die Umgestaltung von Plätzen zuständig, die zur beschleunigten Gentrifizierung mehrerer Stadtteile beitrug. In ihrem Portfolio sind auch einige ambitionierte Spielplätze in wohlhabenderen Gegenden. Diese zielen darauf ab, die Lebensqualität der Bewohner_innen - oder jener, die es werden wollen - zu verbessern. Weitläufige Flächen mit freier Sicht, viel Licht und etlichen Überwachungskameras sorgen dafür, dass unerwünschte Gruppen (Obdachlose, informelle Straßenhändler_innen) nicht hierherkommen.

„Eines der großen Probleme, die es [vor der Umgestaltung des Puschkin-Parks] gab, waren die Unebenheiten, erinnern Sie sich? Es handelte sich um eine Reihe von Plattformen, die sich überlappten, dienass wurden oder über die Kinder stolpern konnten. DieSpielgeräte waren in einem schrecklichen Zustand, es gab eine Rutsche, auf der 


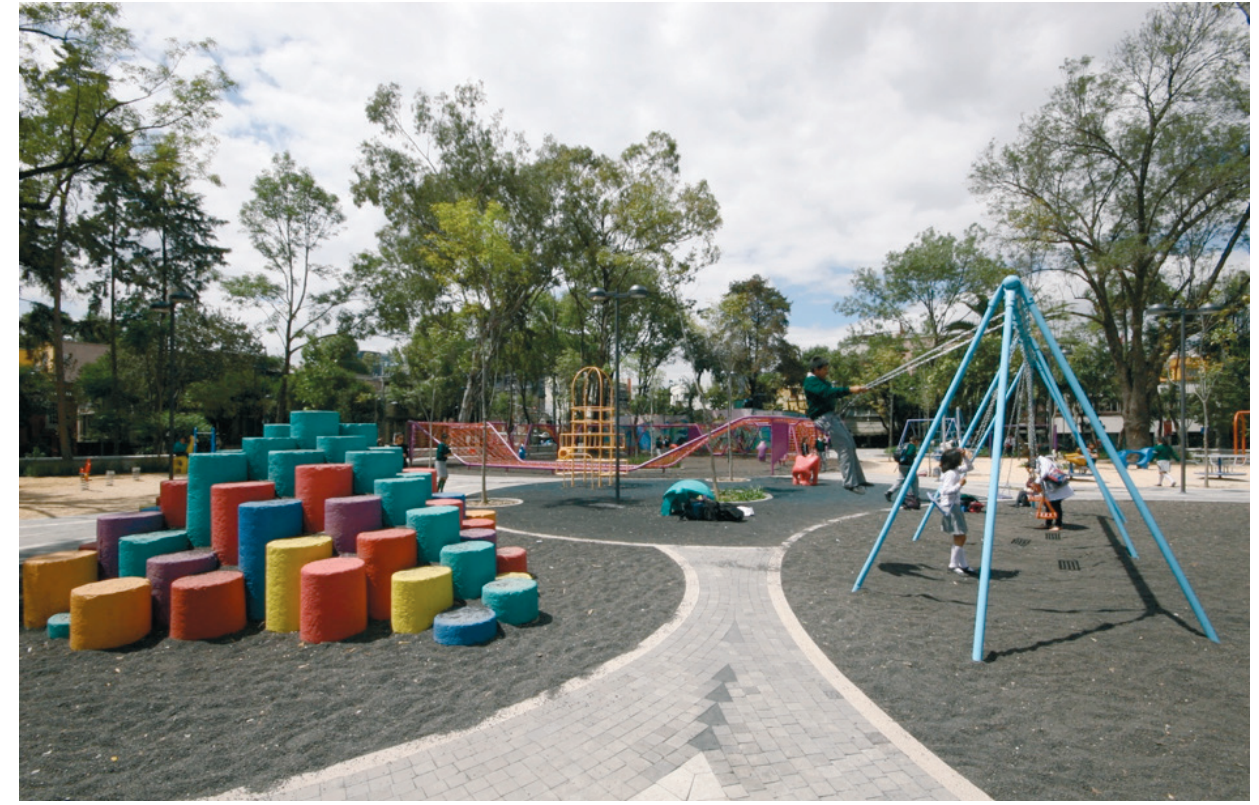

sich J ugendliche zum Rauchen und Drogenkonsum aufhielten, sie waren also eine Infektionsquelle. [...] Die Rutsche wurde abgerissen, weil sie ein von den Bewohnern selbst erkannter Brennpunkt sozialer Problemewar, deshalb haben sie darum gebeten, siezu entfernen."[13]

„Bei [den umgestalteten Parks] handelt es sich umbereits existierende, verlassene Räume, die wie gesagt von Landstreichern, von Delinquenten besetzt waren. Sie werden instand gesetzt, um diese Leute auszurotten."[14]

Die Rolle von Spielplätzen im Prozess der zunehmenden räumlichen Fragmentierungvon Stadtteilen kann an den Beispielen des Puschkin-Park (2016 von der AEP umgestaltet; vgl. Abb. 5) und des Dr.-Ignacio-Chávez-Parks (vgl. Abb. 6) verdeutlicht werden. Diese befinden sich gegenüberliegend auf beiden Seiten der Avenida Cuauhtémoc in Mexiko-Stadt. Dieseviel befahreneStraße markiert die Grenze zwischen dem stark gentrifizierten Viertel Roma und dem marginalen, als unsicher geltenden Viertel Doctores. Der Dr.-IgnacioChávez-Park ähnelt mit seinen Spielgeräten aus Plastik, den abgenutzten stoßdämpfenden Bodenbelägen und der wuchernden Vegetation, die die Sicht einschränkt, zahlreichen anderen Spielplätzen in der Stadt.

Derim Puschkin-Park gelegene SpielplatzistTeil der Aufwertungsstrategie des Viertels und bricht völlig mit der Praxis des willkürlichen Aufstellens von Plastikgeräten. Stattdessen wurden die älteren Spielgeräte aus Beton aufwendig restauriert und neue, formschöne Metallspielgeräte wurden ergänzt.[15] Anstelle eines Gummibodens liegen Naturmaterialien aus, die geometrische Formen auf den Boden zeichnen. Die alten Bäume wurden entfernt und durch wenige neue ersetzt - zusammen mit der Beleuchtung garantiert dies eine gute Sichtbarkeit bei Tag und Nacht. Der PuschkinPark wurde - zusammen mit dem Spielplatz - von Architekt_innen und Landschaftsplaner_innen gestaltet, mit dem Ziel, einen öffentlichen Raum zu schaffen, in dem die Hyperüberwachung der Kinder in perfekter Weise mit den ästhetischen Vorlieben der hier ansässigen wohlhabenden Klasse zusammenfällt.
Abb. 5 Jardín Pushkin, Mexiko-Stadt, 2016 (Quelle: Börries Nehe) 
Abb. 6 Jardín Dr. Ignacio Chávez, Mexiko-Stadt, 2020 (Quelle: Ruth Pérez López)

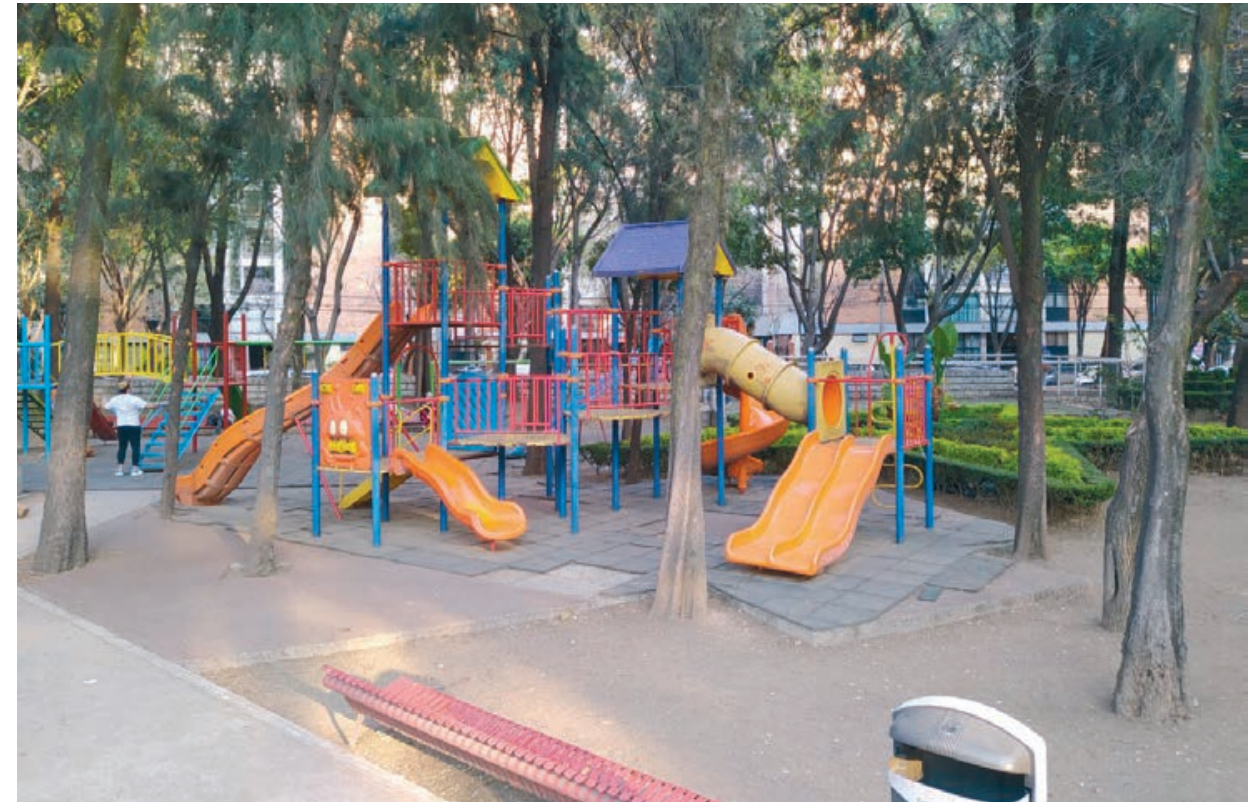

Die hier offenbar werdende klassistische Planung, die sich einerseits in der ungleichen Verteilung öffentlicher Räume in der Stadt und andererseits in der sehr unterschiedlichen Qualität dieser Räume widerspiegelt, verteidigen Beamt_innen der Stadtverwaltung ganz offen:

„[Die Frage ist,] welche Art von Spielgeräten wir brauchen, welche Art von Spielgeräten für verschiedene Arten von Kindern, denn die Kinder aus [dem armen Bezirk] Iztapalapa sind nicht die gleichen wie die Kinder aus [dem reichen] Santa Fe. Sie sind nicht gleich, auch wenn sie auf die gleicheWeise essen. Aber sie haben unterschiedliche Bedürfnisse."[16]

„Nun, wenn man alle Aspekte berücksichtigt..., kommt es auf die Umgebung an, nicht wahr? Das Viertel San Felipe de J ésus in [dem Bezirk] Francisco I. Madero zum Beispiel ist ein sehr konfliktreiches Viertel. Und dann gehen wir in das Viertel Lindavista: Das ist eine andere Art von Viertel. Ich stellein San FelipedeJ esus und Lindavista nicht die gleichen Spielgeräteauf, verstehen Sie? Da werdeich andere Arten von Spielgeräten aufstellen [...], [die] widerstandsfähiger sind, diekampferprobter sind, richtig? Im anderen [Viertel] sind sieteurer. Naja, weil die da drüben, die zerlegen sie, dieklauen sie, die schneiden sieauf, die nehmen kiloweisedavon mit, was weißich. Nun, es kommt auf die Umgebung an, auf viele Dinge."[17]

Spielplätze in wohlhabenden Gegenden und in Vierteln, die einen Prozess der Gentrifizierung durchlaufen, sind gepflegter, doch ebenso wie die Straßen und Plätze „nur Garnitur für großeImmobiliengeschäfte“ (Delgado 2015: 11). Tatsächlich werden einige dieser öffentlichen (und mit öffentlichen Geldern finanzierten) Räume direkt neben großen (privaten) Immobilienprojekten errichtet, wie beispielsweise der "lineare Park“ in Nuevo Polanco (Delgadillo 2014).[18] Somit ist die Aufmerksamkeit, die diesen städtischen Spielplätzen gewidmet wird, weniger einem Interesse am kindlichen Spiel geschuldet als vielmehr Teil städtischer Umstrukturierungsmaßnahmen im Zuge der Gentrifizierung. Die „Ästhetisierung des Viertels“(Smith 1996: 351) 
steigert dessen kommerziellen Wert und stellt eine „Klassenrekonstruktion der innerstädtischen Landschaft" dar: Gentrifizierte Viertel werden so umgestaltet, dass sie dem Lebensstil der Mittelschicht entsprechen. Dazu gehört die „Geringschätzung [...] der Straße zugunsten ihrer Wohn- und Schlafzimmer" (Smith 1996: 87), aber auch die Forderung nach sicheren Räumen für Kinder. Die Schaffung oder Umgestaltung dieser Räume geht zwangsläufig einher mit dem Ausschluss oder der Verweigerung des Zugangs einher „für all die Menschen, die nicht in der Lage sind, die Manieren jener Mittelschicht an den Tag zu legen, für deren Nutzung sie bestimmt sind“ (Delgado 2015: 10).

Diese Räumezeigen nur scheinbar eine „Gemeinschaftlichkeit“städtischer öffentlicher Güter; tatsächlich tragen sie dazu bei, den Wert des Vermögens privilegierter Eigentümer_innen zu steigern. So reduzieren öffentliche Räume, die in Strategien der Immobilienaufwertung eingeschrieben sind, eher die Möglichkeit ihrer gemeinschaftlichen Nutzung, als dass sie diese erweitern (Harvey 2012: 145).

\section{Fazit}

Die Geschichte der Spielplätze in Mexiko ist untrennbar mit jener der Spielplätze in den Vereinigten Staaten und Europa verbunden, wie meine Ausführungen gezeigt haben. Dabei wurde deutlich, dass sich der Bau von Spielplätzen größtenteils an Interessen ausgerichtet hat, die nicht am kindlichen Spiel orientiert sind. Bei den ersten Spielplätzen standen noch moralische Argumente im Vordergrund. Heutzutage haben Sicherheitsbedenken viele Spielplätze in aseptische Orte ohne jeglichen Anreiz zum Spiel verwandelt, während gleichzeitig der Rentabilitätslogik unterworfene stark lokalisierte Aufwertungsprozesse die Schaffung aufwendigerer Spielplätze in wohlhabenden Vierteln befördern.

Spielplätze spiegeln wider, wie eine Gesellschaft Kindheit(en) definiert, auch in Bezug auf soziale Klassen. In diesem Sinne entsprechen die PlastikSpielplätze, diegegenwärtigdiemeisten (mexikanischen) Städteüberschwemmen, ebenso wie alle anderen ausdrücklich dem Kinderspiel gewidmeten Flächen einem globalen Bild von Kindheit bzw. Kinderbetreuung, das in erster Linieauf Risikominimierungabzielt. Dem Wesen des kindlichen Spiels laufen diese Spielplätze deshalb in jeder Hinsicht zuwider.

Freilich gab und gibt es immer wieder Ausnahmen: dort, wo Architekt_innen und Designer_innen am Werke sind, die darauf abzielen, das freie kindliche Spiel zu fördern, und wo der Staat bereit und in der Lage ist, erhebliche Investitionen in den Bau und die Instandhaltung dieser Orte zu tätigen. Zudem gibt es einige Städte(Berlin ist ein gutes Beispiel), dieKindern weiterhin eine größere Autonomie gewähren als andere Städte und die nach wie vor eine große Vielfalt an Spielplätzen bieten, deren Gestaltung es den Kindern ermöglicht, zu experimentieren und Risiken einzugehen.

Spielplätze zeugen von dem Stellenwert, der Kindheit in der Stadt zugeschrieben wird. In diesem Sinne hängt die Forderung nach guten städtischen Orten für Kinder ganz grundsätzlich mit der gesellschaftlichen Forderung nach der Integration von Kindern in das städtische Leben zusammen (Ward 1990 [1978]). Wie Kinder den öffentlichen Raum 
tatsächlich nutzen - nämlich indem sie auf ihren Wegen durch die Stadt Lücken und Zwischenräume suchen und finden -, zeigt uns jedoch, dass die Hyperüberwachung diese Zwischenräume und das kollektive Spiel der Kinder zwar einschränken, aber nicht bändigen kann. Das kindlicheSpiel hat weiterhin jenen subversiven Charakter, der es seitjeher ausmacht.

\section{Übersetzung aus dem Spanischen von Börries Nehe.}

\section{Endnoten}

[1] Diese Forschung entstand im Rahmen eines Postdocs am Institut für Sozialforschung der Nationalen Autonomen Universität von Mexiko (UNAM) (2013-2015) innerhalb des Projekts „Citizenship, urban space and children's social actorship: How to think children's integration in the city?" und des Projekts „Public spaces for children in Mexico City" (Französisches Zentrum für mexikanischeund zentralamerikanische Studien (CEMCA)/ Französische Botschaft) zwischen 2015 und 2017. Sie umfasste die Entwicklung von Workshops zur Untersuchung der Beziehung von Kindern zu Mexiko-Stadt (Gülgönen/ Corona 2015), Interviews mit Beamt_innen und Stadtplaner_innen (Gülgönen/ Laboratorio para la ciudad 2016) und die Produktion des Dokumentarfilms Ciudad Grande (Gülgönen/Álvarez 2017).

[2] Das Projekt des Architekten Mario Pani war damals das größte Wohnungsbauprojekt der Welt: 102 Gebäude mit 12.004 Wohnungen für rund 78.000 Menschen, dazu 600 Geschäfte, 21 Schulen, sechs Gesundheitsstationen und Krankenhäuser, drei Sportzentren, drei Theater und ein Kino. 60 Prozent der Gesamtfläche wurden für öffentliche Räume genutzt. Zahlreiche Orte waren für Kinder gedacht, die „Rakete“war jedoch der imposanteste von ihnen.

[3] Der erste skrammellegeplads (Gerümpelspielplatz) wurde in den 1930er J ahren in Dänemark vom Landschaftsarchitekten Carl Theodor Marius Sørensen geschaffen. Der Begriff „Abenteuerspielplatz“ wurde 1953 eingeführt.

[4] Es handelt sich um die Normen UNE 147103 („Planungund Verwaltungvon Spielplätzen und Parks im Freien“, 2001), UNE-EN 1176 („Ausstattung von Spielplätzen und Spielflächen“, 2009) und UNE-EN 1177 („Stoßabsorbierende Oberflächenbeläge für Spielplätze“", 2009).

[5] Interview, Generaldirektion für städtischeArbeiten und Dienstleistungen der Delegation Iztapalapa, Mexiko-Stadt, 21.4.2016.

[6] Interview, Koordination von Sonderprojekten der Delegation Miguel Hidalgo, 28.3.2016.

[7] Das wiederkehrendeArgument, dass Kinder sich an kaputtem Metall schneiden könnten, ist kaum haltbar, wenn dieselben Befragten angeben, dass Metall und Holzin den eigenen Werkstätten der Gemeindeverwaltung repariert werden können, die Kunststoffbausätze aber mangels Budget nicht ausgetauscht werden, wenn sie kaputtgehen (Interview, Generaldirektion für städtischeArbeiten und Dienstleistungen der Delegation Iztapalapa, Mexiko-Stadt, 21.4.2016).

[8] Interview, Umweltministerium, Direktion „Bosque de Chapultepec“, Mexiko-Stadt, 5.4.2016.

[9] Interview, Koordination von Sonderprojekten der Delegation Miguel Hidalgo, 28.3.2016.

[10] Interview, Generaldirektion für städtischeArbeiten und Dienstleistungen der Delegation Iztapalapa, Mexiko-Stadt, 21.4.2016.

[11] Pocket parksin Mexiko-Stadt sind zwischen 100 und 400 qm groß, während siein London bis zu 4.000 qm umfassen (Parques de México 2014; Ministry of Housing, Communities \&Local Government o. J .).

[12] Interview, Generaldirektion für städtischeArbeiten und Dienstleistungen der Delegation Iztapalapa, Mexiko-Stadt, 21.4.2016.

[13] Interview, Behörde für den öffentlichen Raum, 4.5.2016. 
[14] Interview, Generaldirektion für städtischeArbeiten und Dienstleistungen der Delegation Iztapalapa, Mexiko-Stadt, 21.4.2016.

[15] Der Weg, der hier mit der Instandsetzung alter Spielgeräte eingeschlagen wurde, ist neu: „Also was wir getan haben, war einerseits zu versuchen, bestimmte Elemente wiederaufzunehmen und die Verwurzelung und das historische Gedächtnis einiger Spielelemente anzuerkennen, wie zum Beispiel die Betontiere, die sehr traditionelle Spielgeräte in den Parks sind, die in den 1970erJ ahren entwickelt wurden. Diese Spiele wollten wir würdigen, wiederherstellen und sanieren, damit sienicht aus dem Gedächtnis und der städtischen und sozialen Landschaft verschwinden. Und wir haben viele der bestehenden Spielgeräte instandgesetzt. “(Interview, Behörde für den öffentlichen Raum, 4.5.2016)

[16] Interview, Dirección del Patrimonio Cultural Urbano de la Secretaría de Desarrollo Urbano y Vivienda (SEDUVI), 3.5.2016.

[17] Interview, Generaldirektion für städtischeArbeiten und Dienstleistungen der Delegation Iztapalapa, Mexiko-Stadt, 21.4.2016.

[18] Mit dem Regierungswechsel in Mexiko-Stadt im J ahr 2018 wurde die AEP aufgelöst. Die aktuelle Gouverneurin von Mexiko-Stadt kritisierte in diesem Zuge die Ausrichtung der Vorgängerregierung scharf und hob insbesondere die Tatsache hervor, dass „[der] städtische Raum nichtim Dienst der Interessen der Immobilienentwickler, der Korruption der Gouverneure und der Planung mit externen Programmen stehen kann, die das Recht der Bürger auf partizipative Planung ihrer Nachbar_innenschaften, Viertel und Städte nicht anerkennen“" (Gobierno de la Ciudad de México 2019).

\section{Autor innen}

Tuline Gülgönen arbeitet über das Verhältnis von Kindern zur Stadt in kollaborativen interdisziplinären Projekten und untersucht, wie man ihren Blick auf dieWelt durch hybride und manchmal experimentelle Methoden verstehen kann.

tulinegulgonen@gmail.com

\section{Literatur}

Ansell, Nicola (2019): Global south research in children's geographies. From useful illustration to conceptual challenge. In: Tracey Skelton / Stuart Aitken (Hg.), Establishing geographies of children and young people. Singapur: Springer, 51-70.

Ariès, Philippe (1993): Essais de mémoire 1943-1983. Paris: Editions du Seuil.

Benjamin, Walter (1969): Kulturgeschichte des Spielzeugs. In: Walter Benjamin, Über Kinder, J ugend und Erziehung. Frankfurt am Main: Suhrkamp, 61-65.

Borja, J ordi (2010): La ciudad conquistada. Madrid: Alianza Editorial.

Borja-Villel, Manuel J . / Díaz, Tamara / Velázquez, Teresa / Museo Nacional Centro de Arte Reina Sofía (Hg.) (2014): Playgrounds. Reinventar la plaza. Madrid: Museo Nacional Centro de Arte Reina Sofía/ Siruela.

Burkhalter, Gabriela (2018): The playground project. Zürich: J RP Ringier.

Caillois, Roger (2012 [1958]): Les jeux et les hommes. Le masque et le vertige. Paris: Gallimard.

Caldeira, Teresa Pires do Rio (2007): Ciudad de muros. Barcelona: Gedisa.

Capron, Guénola (2012): Sentiment d'insécurité et inconfort chez les classes moyennes et supérieures des banlieues résidentielles au Sud et au Nord. In: Espaces et sociétés 150/2, 129-147.

Carver, Alison / Watson, Ben / Shaw, Ben / Hillman, Mayer (2013): A comparison study of children's independent mobility in England and Australia. In: Children's Geographies 11/4, 461-475.

Cazalis, Ferdinand (2018): Le gouvernement des playgrounds. Histoire fragmentée des aires de jeux, 1770-2010. In: Clément Cogitore (Hg.), Encore un jour pour le poisson-rêve. Another banana day for the dream-fish. Paris: Palais de Tokyo, 167-189. 
Delgadillo, Víctor (2014): La política del espacio público y del patrimonio urbano en la Ciudad de México. Discurso progresista, negocios inmobiliarios y buen comportamiento social. Paper präsentiert beim XIII. Coloquio Internacional de Geocrítica. „El control del espacio y los espacios de control", 5.-10.5.2014, Barcelona.

Delgado, Manuel (2015): El espacio público como ideología. Madrid: Catarata.

van Eyck, Aldo (2014 [1962]): Sobreel diseño del equipamiento lúdico y la disposición delos espacios de juego. In: Manuel J. Borja-Villel / Tamara Díaz / Teresa Velázquez / Museo Nacional Centro de Arte Reina Sofía (Hg.) (2014), Playgrounds. Reinventar la plaza. Madrid: Museo Nacional Centro de Arte Reina Sofía/ Siruela, 120-161.

Giglia, Angela (2003): Espacio publico y espacios cerrados en la Ciudad de México. In: Patricia Ramírez Kuri (Hg.), Espacio público y reconstrucción de ciudadanía. MexikoStadt: FLACSO-Porrua, 341-364.

Gillis, John R. (2018): L'insularisation des enfants. Remodeler les paysages mythiques de l'enfance. In: Clément Cogitore (Hg.), Encore un jour banane pour le poisson rêve. Another banana day for the dream-fish. Paris: Palais de Tokyo, 135-147.

Gobierno de la Ciudad de México (2019): Programa de gobierno de la Ciudad de México 2019-2024. https:/ / plazapublica.cdmx.gob.mx/ processes/ programa-de-gobiernocdmx/f/1/proposals/46 (letzter Zugriff am 16.7.2021).

Guerrien, Marc (2001): Délinquance, criminalité et sentiment d'insécurité. Quelques réflexions sur le cas de Mexico. In: Cahiers des Amériques latines 37, 61-84.

Gülgönen, Tuline (2021): The notion of neighborhood. Children's perspectives on the city and sense of place in Mexico City. In: Victoria Derr / Yolanda Corona (Hg.), Latin American transnational children and youth. Experiences of nature and place, culture and care across the Americas. New York: Routledge, 39-54.

Gülgönen, Tuline / Álvarez, Ana (2017): Ciudad grande. CEMCA.

Gülgönen, Tuline / Corona, Yolanda (2015): Children's perspectives on their urban environment and their appropriation of public spaces in Mexico City. In: Children, Youth and Environments 25/2, 208-228.

Gülgönen, Tuline / Corona, Yolanda (2019): ¿Jugar en la ciudad? La percepción de niñas y niños de la Ciudad de México sobre su entorno urbano. In: Cadernos de Pesquisa em Educação PPGE/UFES 21/49, 60-80.

Gülgönen, Tuline / Laboratorio para la ciudad (2016): Jugar la ciudad. Reimaginar los espacios públicos urbanos dejuego para la infancia en la Ciudad de México. Mexiko-Stadt: CEMCA/ Laboratorio para la ciudad.

Hanson, Karl / Abebe, Tatek / Aitken, Stuart C. / Balagopalan, Sarada / Punch, Samantha (2018): "Global/local“ research on children and childhood in a "global society". In: Childhood 25/3, 272-296.

Hart, Roger (1986): The changing city of childhood. Implications for play and learning. The 1986 Catherine Molony memorial lecture. New York: City College Workshop Center.

Hart, Roger (2002): Containing children. Somelessons on planning for play from New York City. In: Environment and Urbanization 14/ 02, 135-148.

Harvey, David (2012): Rebel cities. From the right to the city to the urban revolution. London/ New York: Verso.

Hillman, Mayer / Adams, John / Whitelegg, John (1993): One false move. A study of children's independent mobility. London: Policy Studies Institute.

Holloway, Sarah (2014): Changing children's geographies. In: Children’s Geographies 12/4, 377-392.

Holloway, Sarah / Valentine, Gill (2000): Children's geographies. Playing, living, learning. London: Routledge.

Huizinga, J ohan (1951): Homo ludens. Essai sur la fonction social du jeu. Paris: Gallimard.

Jacobs, J ane (1992 [1961]): The death and life of great American cities. New York: Vintage Books.

Jansson, Märit (2009): Management and use of public outdoor playgrounds. Doctoral Thesis. Alnarp: Swedish University of Agricultural Sciences.

Karsten, Lia / van Vliet, Willem (2006): Children in the city. Reclaiming the street. In: Children, Youth and Environments 16/1, 151-167.

Katz, Cindy (2006): Los terrores de la hipervigilancia. Seguridad y nuevas espacialidades de la niñez. In: Documents D’Anàlisi Geogràfica 47, 15-29. 
Kyttä, Marketta / Hirvonen, Jukka / Rudner, J ulie/ Pirjola, Iiris / Laatikainen, Tiina (2015): The last free-range children? Children's independent mobility in Finland in the 1990s and 2010s. In: J ournal of Transport Geography 47, 1-12.

Lehman-Frisch, Sonia / Authier, Jean-Yves / Dufaux, Frédéric (2012): „Draw me your neighbourhood“. A gentrified Paris neighbourhood through its children's eyes. In: Children's Geographies 10, 17-34.

Lynch, Kevin (1977): Growing up in cities. Cambridge/ Paris: M.I.T. Press/ UNESCO.

Matthews, Michael Hugh (1992): Making sense of place. Children's understanding of largescale environments. Harmondsworth: Harvester Wheatsheaf.

McKendrick, John H. / Bradford, Michael G. / Fielder, Anna V. (2001): Kid customer? Commercialization of playspace and commodification of childhood. In: Sage Family Studies Abstracts 23/ 1, 3-135.

Ministry of Housing, Communities and Local Government (o. J.): Pocket parks plus: Frequently Asked Questions. https://assets.publishing.service.gov.uk/government/ uploads/system/uploads/attachment_data/file/767523/PPP_Q_A.pdf (letzter Zugriff am 16.7.2021).

Moore, Robin C. (2019 [1986]): Childhood's domain. Play and place in child development. London/ New York: Routledge.

Murnaghan, Ann Marie F. (2019): Play and playgrounds in children's geographies. In: Tracey Skelton / Stuart Aitken (Hg.), Establishing geographies of children and young people. Singapur: Springer. https://link.springer.com/ referenceworkentry/ 10.1007/978 -981-4585-88-0_12-1 (letzter Zugriff 5.10.2021).

Pansters, Wil / Berthier, Héctor Castillo (2007): Violencia e inseguridad en la ciudad de México. Entre la fragmentación y la politización. In: Foro Internacional 47/3 (189), 577-615.

Parques de México (2014): Reciclan espacios con „Pocket Parks“ en el DF. http://www. parquesdemexico.org/consultora/ reciclan-espacios-con-pocket-parks-en-el-df (letzter Zugriff am 16.7.2021).

Pérez López, Ruth (2016): Vivre et survivre à Mexico. Paris: Editions Karthala.

Rasmussen, Kim (2004): Places for children - children's places. In: Childhood 11/3, 155-173.

Rasmussen, Kim/ Smidt, Soren (2003): Children in theneighbourhood. Theneighbourhood in the children. In: Pia Christensen / Margaret O’Brien (Hg.), Children in the city home, neighbourhood and community. London/ New York: Routledge Falmer, 82-100.

Rissotto, Antonella / Giuliani, M. Vittoria (2006): Learning neighbourhood environments. The loss of experience in a modern world. In: Christopher Spencer / Mark Blades (Hg.), Children and their environments. Cambridge: Cambridge University Press, 75-90.

Rodrigues Breitman, Miriam (1994): La construcción social de la infancia delincuente. In: Nueva Sociedad 129, 152-163.

SEDESOL - Secretaría de Desarrollo Social (2007): Guía de diseño del espacio público seguro, incluyente y sustentable. Mexiko-Stadt: SEDESOL.

Smith, Neil (1996): The new urban frontier. Gentrification and the revanchist city. New York: Routledge.

Solano Rojas, Aldo (2018): Playgrounds del México moderno. Mexiko-Stadt: Promotora Cultural Cubo Blanco.

Sosenski, Susana (2018): Infancia y violencia. Los robachicos en las historietas para adultos en México (1945-1950). In: Humanidades. Revista de la Universidad de Montevideo 4, 103-128.

Tarragoni, Federico (2015): J eu, matérialité et politique chez Walter Benjamin. In: Tracés 28, 137-154.

Tonucci, Francesco (1997): La ciudad de los niños. Un modo nuevo de pensar la ciudad. Madrid: Fundación Germán Sánchez Ruipérez.

Tyagi, Megha / Raheja, Gaurav (2020): Indian parents' perception of children's independent mobility in urban neighbourhoods. A case study of Delhi. In: Children's Geographies 19/4, 1-17.

Valentine, Gill / McKendrick, John (1997): Children's outdoor play. Exploring parental concerns about children's safety and the changing nature of childhood. In: Geoforum 28/2, 219-235.

Ward, Colin (1961): Adventure playground. A parable of anarchy. London: Freedom Press. Ward, Colin (1990 [1978]): The child in the city. Harmondsworth: Penguin.

Wridt, Pamela J. (2004): An historical analysis of young people's use of public space, parks and playgrounds in New York City. In: Children, Youth and Environments 14/ 1, 86-106. 
From children's domains to the kingdom of norms. Reflections on playgrounds in Mexico City and global perspectives

The transition from a city-as-playground, appropriated and lived by children, to children's withdrawal into playgrounds specifically designed for them during the20th Century, has been shown in a variety of urban contexts around theworld. Thecurrent proliferation of children's playground equipment of similar design and materials in many cities corresponds to a representation of both childhood and thecity. This paper adopts an interdisciplinary approach to discuss these representations of childhood and the city, and theinteraction between them. With a particular focus on playgrounds in Mexico City, it interrogates their link with the conformation of a global image of childhood. It examines whether the construction of playgrounds aims to enhance children's play, and whether they still constitute spatial references and territories for contemporary children. It critically addresses the concept of public space to show how it can be used to enhancea neoliberal approach to the city. 
